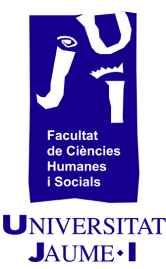

\title{
La enseñanza de las locuciones como recurso lingüístico en los textos de Bachillerato
}


I. Resumen

\begin{abstract}
l. Resumen
El objetivo de este trabajo es analizar la presencia de las locuciones en los manuales de Lengua Castellana y Literatura de 1은 de Bachillerato, así como conocer qué tipo de actividades se proponen en ellos. En primer lugar, se explica la metodología y la descripción del corpus, el cual posibilita la observación y la extracción de los resultados obtenidos de forma objetiva. En segundo lugar, se exponen las características formales más relevantes de las locuciones y la clasificación establecida en el Manual de la Nueva gramática de la lengua española (2010). Seguidamente, se compara su enseñanza en la Educación Secundaria Obligatoria con la del aula de Español como Lengua Extranjera, dado que esta última sitúa las locuciones en una posición más relevante dentro del currículo.
\end{abstract}

Palabras clave: educación, aprendizaje, lengua española, fraseología, locuciones, recurso lingüístico.

\title{
II. Introducción
}

El objeto de estudio de este trabajo es el tratamiento de las locuciones en los manuales de Lengua Castellana y Literatura de 10 de Bachillerato, en los que la enseñanza de este tipo de expresiones fijas apenas tiene cabida. Además, en los niveles superiores, como es este caso, todavía son, como señala Martínez Marín (2000, 328), «insuficientemente atendidas en la enseñanza del castellano, aunque tienen una gran importancia en su estructura y funcionamiento».

Por el contrario, en la enseñanza de español como lengua extranjera, tanto las locuciones como otras unidades pertenecientes a la fraseología son una parte fundamental del currículo, porque sin ellas los alumnos no entenderían muchas de las expresiones que se utilizan con frecuencia en las conversaciones. Asimismo, cada vez más manuales que integran y trabajan las locuciones. Por ejemplo, se han publicado obras como Locuciones y refranes para dar y tomar. El libro para aprender más de 120 locuciones y refranes del español, en el que se destaca la importancia de «trabajar las locuciones de manera integrada en el resto de contenidos del currículo, de modo que no queden aisladas y descontextualizadas» (Gómez González y Ureña Tormo 2015, 3).

Sin embargo, sea como fuere, la poca presencia que adquieren las locuciones en el aula de Secundaria conlleva un empobrecimiento en la competencia comunicativa y cultural de los jóvenes, quienes se ven obligados, en muchos casos, a emplear proformas o palabras baúl en vez de otros elementos lingüísticos como las locuciones, que 
les permitirían dar un notable salto cualitativo y enriquecer con numerosos matices el mensaje (Núñez-Román 2015, 156). Estas expresiones tienen, de algún modo, una belleza especial que hace que recurra a ellas cuando se quiere decir más de lo que se está diciendo, dado que, evidentemente, no se expresa con la misma fuerza ilocutiva llueve mucho que llueve a cántaros. Es por eso por lo que algunos investigadores «sugieren que quizá es la gramática tal y como se enseña lo que no ayuda a los alumnos a mejorar los usos» (Fontich 2014, 5). En definitiva, se trata de dejar constancia de la poca claridad a la hora de trabajar este aspecto lingüístico que, en muchos casos, queda casi invisibilizado en el aula.

\section{Objetivos}

El objetivo principal es conocer cuál es el lugar que ocupan las locuciones en los manuales de texto y, así, entender cómo se lleva a la práctica su enseñanza en el aula. Por eso, consideramos importante analizar y comparar el tratamiento de las locuciones en los manuales de Lengua Castellana y Literatura de 10 de Bachillerato a través de un cuestionario general para identificar qué clases de locuciones se explican y si se contextualizan dentro de un texto.

\section{Las locuciones como elemento didáctico}

\subsection{Sobre el nombre y los rasgos generales de las locuciones:}

El concepto de locución aparece explicado en los manuales analizados como «expresión pluriverbal», «expresión fija», «expresión fraseológica», "combinación de dos o más palabras», etc. Todas estas definiciones coinciden en que se trata de un conjunto de palabras que forman expresiones fijas y estables o, dicho de un modo mejor:

Son unidades léxicas formadas por más de dos palabras gráficas en su límite inferior, cuyo límite superior se sitúa en el nivel de la oración compuesta. Dichas unidades se caracterizan por su alta frecuencia de uso, y de coaparición de sus elementos integrantes; por su institucionalización entendida en términos de fijación y especialización semántica; por su especificidad idiomática; así como por el grado en el cual se dan todos estos aspectos en los distintos tipos. (Corpas Pastor 1996, 20)

Pero ¿cómo sabemos que estamos ante locuciones y no otro tipo de expresiones fraseológicas como las combinaciones libres? Zuluaga $(1980,136)$ explica que mantienen siempre un mismo orden sin que se permita la alteración como en las combinaciones libres; rechazan la inserción de otras palabras que no formen parte de la 
propia combinación, como tampoco permiten separar o modificar el género y número de los propios elementos integrantes. Además, son idiomáticas o semiidiomáticas y esto implica que - por lo generalno se pueden traducir, porque el significado global de la expresión no es la suma de sus componentes.

Las locuciones pueden cumplir la función de diferentes tipos de palabras y, por eso, el Manual de la Nueva gramática de la lengua española $(2010,222-633)$ las clasifica en siete tipos:

- Locuciones nominales: pez gordo

- Locuciones adjetivales: en jarras

- Locuciones verbales: meter la pata

- Locuciones adverbiales: ni más ni menos

- Locuciones prepositivas: a base de

- Locuciones conjuntivas: pese a que

- Locuciones interjectivas: iNi en broma!

\subsection{Clasificación de las locuciones en los distintos manuales}

Para poder determinar cómo se trabajan las locuciones en los manuales, se ha analizado la frecuencia de aparición de cada uno de los tipos descritos anteriormente. De este modo, obtenemos los siguientes porcentajes:

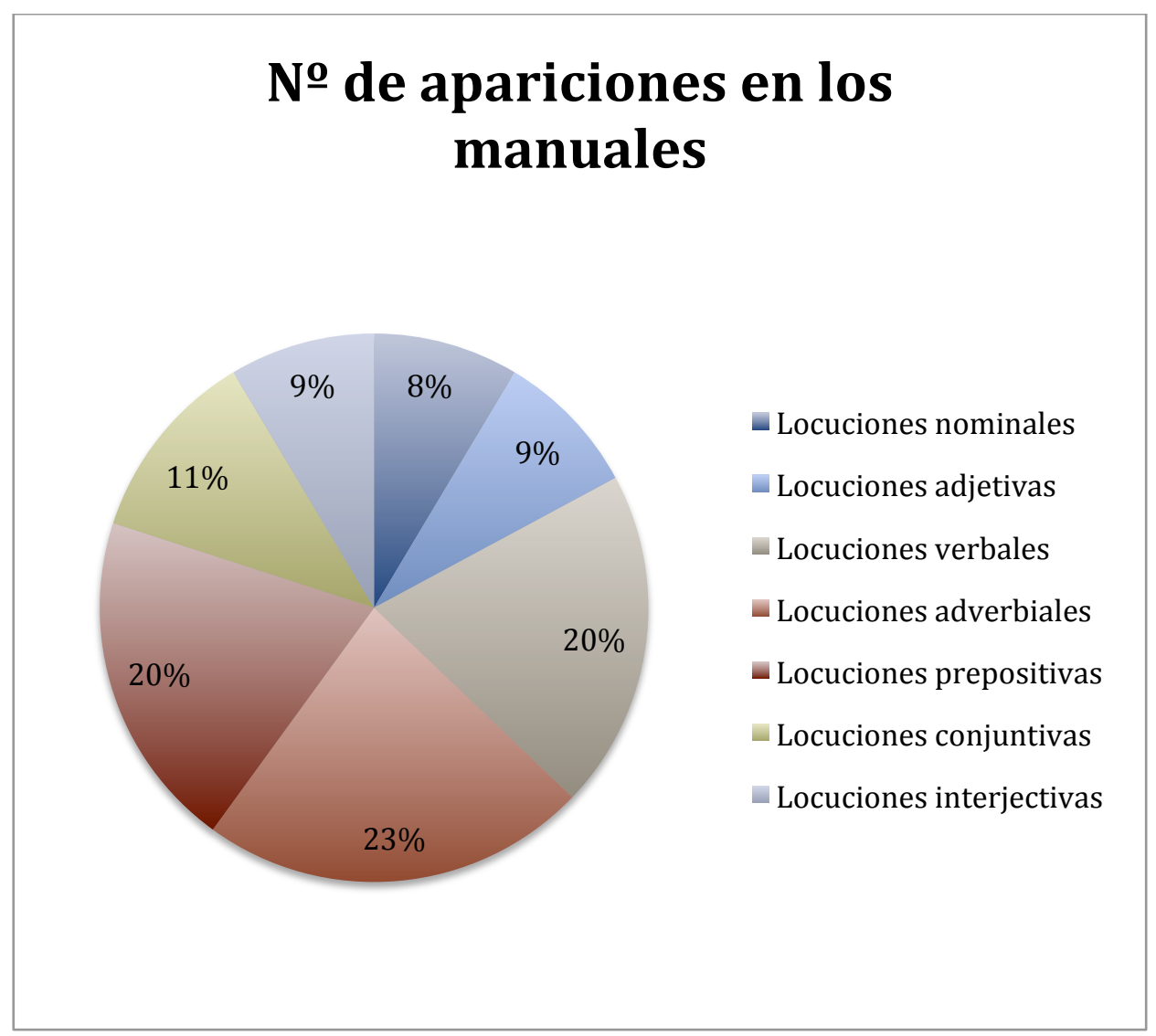

Figura 1. Aparición de los tipos de locuciones (elaboración propia) 
Por lo que respecta a las locuciones nominales, son las menos populares en los manuales didácticos de Bachillerato, dado que tan solo aparecen un $8 \%$ de las veces y, en la mayoría de los casos, se hace de forma general. Esto tal vez se deba a la dificultad que entraña establecer una separación entre los grupos nominales y las locuciones nominales.

Por su parte, las locuciones adjetivas también constituyen un grupo bastante olvidado en los manuales, puesto que apenas llegan al $9 \%$. La razón puede ser la confusión que presenta su forma frente a sintagmas preposicionales de composición libre.

En cambio, las locuciones verbales conforman uno de los grupos más incluidos en los manuales. Además, en buena parte de los casos, las explicaciones de estos sintagmas fijos se ofrecen por oposición a las perífrasis verbales, por lo que resulta mucho más fácil la visualización e identificación de este tipo de locuciones. Se sitúan, junto con las locuciones prepositivas, en un $20 \%$.

Las locuciones adverbiales son las más estudiadas en los manuales ( $23 \%$ ); tal vez sea por la facilidad que presentan a la hora de identificarlas o de sustituirlas por un adverbio.

De igual manera, las locuciones preposicionales se sitúan entre las más explicadas en los manuales con un $20 \%$. Existe una gran variedad de esquemas que forman locuciones prepositivas $y$, además, como se afirma en el manual de la $\operatorname{NGLE}(2010,560)$, "permiten gramaticalizar significados mucho más específicos que los que designan las preposiciones simples».

El grupo de las locuciones conjuntivas, que se incluye junto con las conjunciones, logra alcanzar un $11 \%$ en el análisis.

Finalmente, las locuciones interjectivas se sitúan con un $9 \%$ en el análisis.

Estos porcentajes responden de forma negativa a la pregunta 5 (¿Se dicen todos los tipos de locuciones que existen en la lengua española?) del cuestionario utilizado para el análisis de manuales. A continuación, por ofrecer un ejemplo, vemos cómo ilustra esta cuestión el manual de la editorial Micomicona (2015):

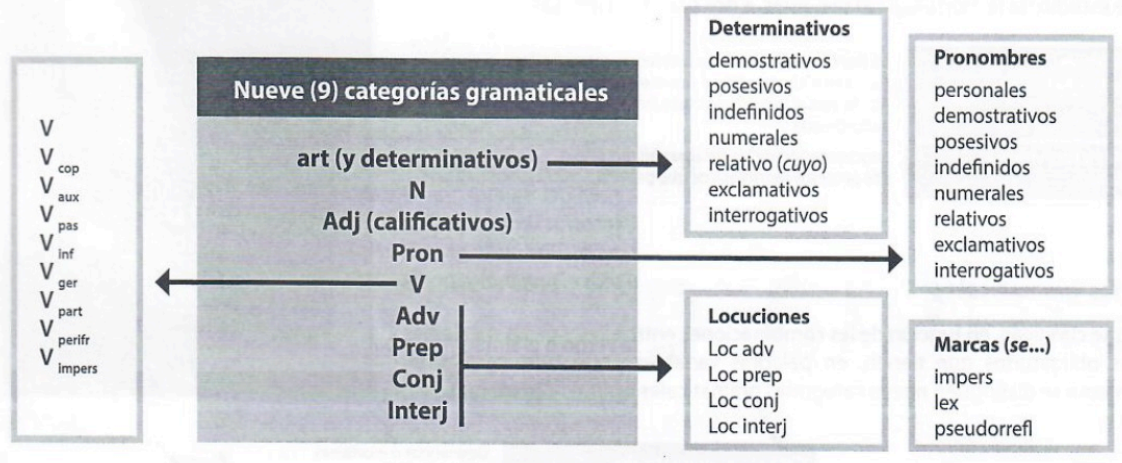

Figura 2. Esquema del manual Micomicona 
Como podemos observar, solo las categorías gramaticales del adverbio, preposición, conjunción e interjección poseen locuciones, mientras que del resto no se dice nada. En definitiva, se trata de un reflejo de lo que encontramos en la mayoría de los manuales, los cuales emplean una clasificación incompleta de las locuciones que se han descrito al inicio de este apartado.

Además, en ninguno de ellos aparece una explicación justificada de por qué se han elegido unas determinadas locuciones y no otras, como tampoco se explica la ausencia de los grupos no tratados. Esto conlleva una confusión a la hora de aprender y reconocer la lengua, porque en los textos no se discrimina la aparición y el uso de unas u otras locuciones. Por lo tanto, la pregunta que nos podemos plantear es la siguiente: ¿por qué a las locuciones no se les concede la misma importancia que al resto de las categorías gramaticales si se encuentran en nuestro día a día?

\subsection{Del uso inconsciente al uso consciente}

El aprendizaje y el uso de las locuciones se adquieren de forma natural e inconsciente. Diversas hipótesis psicolingüísticas como la de Belinchón $(1999,354)$ exponen que los niños empiezan a conocer la estructura del lenguaje a partir de los cuatro años y medio. Al mismo tiempo, «los niños van aprendiendo progresivamente las estructuras fijas de la lengua, y lo hacen del mismo modo que van asimilando otros mecanismos del lenguaje, como las formas flexivas de los verbos» (Ruiz Gurillo 2001, 89).

De esta forma, aprenden las estructuras de un gran número de locuciones y expresiones fraseológicas, como también empiezan a conocer el significado y los contextos en los que se pueden utilizar. Sin embargo, todo esto es producto de la adquisición de la lengua de forma natural y progresiva, pero esto no implica que sepan qué tipo de expresiones están utilizando ni, en un nivel más complejo, las funciones que dichas unidades fraseológicas pueden tener en textos escritos. De hecho, "varias experiencias e investigaciones indican que, para aprender a comunicarnos de manera eficiente, es esencial que las actividades de producción y de recepción de textos de diversos tipos se acompañen de actividades de reflexión sobre los usos mismos» (Fontich 2014, 5).

Por todo ello, las locuciones, como recurso lingüístico que son, deberían introducirse en todos los niveles de la Educación Secundaria, y explicarse con más detalle en Bachillerato. El cambio de un uso inconsciente de las estructuras fraseológicas al uso consciente no es un proceso sencillo; por tanto, previamente, deben trabajarse las bases teóricas para dejar bien definido qué son las locuciones.

Así pues, debemos trabajar en el uso consciente de aquellos recursos que, en el caso de las locuciones, se han adquirido de forma 
inconsciente, para que tengan un sentido y una finalidad dentro del

discurso. De esta manera, saben combinarlas con otras unidades lingüísticas para conseguir el propósito lingüístico que tiene el texto (persuadir, exponer, describir, informar, etc.).

\subsection{Tratamiento a partir de textos}

\subsubsection{Recurso de cohesión y coherencia}

Si partimos del texto como unidad de análisis, observamos la convergencia de diversos fenómenos lingüísticos presentes en la lengua en uso. Aparecen de forma recurrente para que los textos funcionen bien cohesionados y sean coherentes. En este apartado observaremos cómo las locuciones "contribuyen en especial a la cohesión de un texto y, en consecuencia, a proporcionarle su unidad y coherencia» (Ruiz Gurillo 2001, 66).

A continuación, nos serviremos de un ejemplo extraído de la obra de Ruiz Gurillo $(2001,67)$ :

Sara: No, no, no, porque mi novio no se lo merece. Por una parte, ya me ha engañado suficientes veces; por otra parte, nunca tiene ni un detalle conmigo. Sin embargo, es una persona maravillosa con los demás. De todas maneras, no pienso seguir así [...]. Por supuesto que lo quiero, a pesar de que la situación en la que estamos es ya insostenible.

Como vemos en este ejemplo, las locuciones configuran el esqueleto del texto. Podríamos cambiar el propósito de la conversación, pero, si mantenemos las locuciones, veremos que el significado se dirige hacia la misma dirección. Esto nos permite observar que el cuerpo de un texto necesita estar cohesionado gracias a los recursos lingüísticos que intervienen. En esta línea, las locuciones nos ayudan a organizar el mensaje y a extraer la idea de la que se trata en el ejemplo. Al mismo tiempo, estas unidades fijas también se ponen al servicio de los hablantes para satisfacer los propósitos a los que se quiere llegar. Por consiguiente, las locuciones también sirven «como armas argumentativas» (Ruiz Gurillo 2001, 65), porque permiten reforzar las ideas o debilitar los argumentos del otro interlocutor.

En definitiva, nos encontramos ante elementos lingüísticos que pueden trabajarse en el aula como recursos de coherencia y cohesión, como armas argumentativas para reforzar nuestras opiniones, etc. Por lo tanto, para lograr entender su uso lingüístico deben enseñarse dentro de un contexto determinado, dado que pueden adquirir diversas funciones según la finalidad del texto. 


\subsubsection{Recurso expresivo}

El discurso coloquial está repleto de locuciones nominales, adjetivales, verbales, adverbiales e interjectivas, por lo que este puede servir de fuente para el estudio de las locuciones. Como afirma Ruiz Gurillo (2001, 72):

El lenguaje coloquial es [...] una fracción o nivel de lenguaje total que se destaca por su carácter pintoresco reflejado en multitud de expresiones y vocablos intraducibles a otros idiomas, fundados muchas veces en alusiones metafóricas.

Cuando hablamos de expresiones y vocablos intraducibles, nos referimos a unidades fraseológicas como las que se estudian en este trabajo, las cuales son capaces de ocupar el lugar del léxico común. A continuación, presentamos un ejemplo de conversación coloquial que ha sido extraído del corpus del grupo Val.Es.Co:

L: no/ era una chica de quinto/ a mí la verdad es que me importaba muy poco lo que ella hiciera ME DABA igual ¿no? Lo que pasa que me quedé/ un poco de piedra [...].

En este ejemplo, el interlocutor emplea la locución adjetiva de piedra para expresar su sorpresa ante tal situación. A pesar de que no se puede hablar de un léxico estrictamente coloquial, es cierto que aparecen con frecuencia ciertas frases y expresiones como llover a cántaros, libro abierto, no ver tres en un burro, etc. Estos ejemplos nos muestran un registro más relajado, propio del lenguaje coloquial.

Ciertamente, se pueden trabajar las locuciones desde las diferentes tipologías textuales si se conocen las funciones que tienen en cada texto. Por tanto, es necesario abordarlas desde un enfoque comunicativo que nos ayude a comprender su uso pragmático en cada texto. No obstante, se recomienda que se mencionen e integren de forma transversal durante todo el curso a partir de los textos que se estudian en el aula, en vez de tratarlas en una sola unidad didáctica, dado que el uso y la aparición de las locuciones sería más forzoso y artificial.

\section{Metodología del análisis}

\subsection{Selección del corpus recogido}

Este trabajo se basa en el análisis de diez manuales de texto de Lengua Castellana y Literatura utilizados en el curso de 10 de Bachillerato. La selección de este curso para el estudio que se va a realizar se basa en que el alumnado de este nivel debería conocer todas las partes integrantes de las categorías gramaticales. Además, 
el currículo establece que los estudiantes tienen que saber «aplicar sistemáticamente los conocimientos sobre las distintas categorías gramaticales en la realización, autoevaluación y mejora de los textos orales y escritos, tomando conciencia de la importancia del conocimiento gramatical para el uso correcto de la lengua» (Real Decreto 1105/2014).

A continuación, se presenta una tabla que muestra el listado de manuales que configura el corpus:

Tabla 1. Manuales del corpus (elaboración propia)

\begin{tabular}{|cc|}
\hline Editorial & Año \\
\hline Santillana & 2016 \\
\hline Anaya & 2015 \\
\hline Casals & 2015 \\
\hline Editex & 2015 \\
\hline Micomicona & 2015 \\
\hline Oxford & 2015 \\
\hline Sansy & 2015 \\
\hline Teide & 2009 \\
\hline Castellnou & 2008 \\
\hline McGraw-Hill & 2007 \\
\hline
\end{tabular}

Como se observa en la tabla, se han escogido manuales de diferentes editoriales para analizar y comparar el tratamiento de las locuciones en cada uno de ellos. En los apartados siguientes se explica cómo se ha realizado este proceso.

\subsection{Procedimiento de análisis}

La metodología empleada para el análisis de los manuales de texto se lleva a cabo a través de un cuestionario en el que se especifican las preguntas.

Con este cuestionario se puntúa de 0 a 4 la aparición y el tratamiento de las locuciones en todos los manuales. Al mismo tiempo, se han asociado diferentes colores a cada uno de estos valores numéricos. De esta forma, tenemos, por un lado, el análisis cuantitativo que responde al valor numérico que se obtiene de la suma de puntos tras responder a todas las preguntas; por otro lado, los colores vinculados a los números se asocian a un análisis cualitativo.

Posteriormente, se ofrece una tabla para ilustrar la explicación anterior sobre el análisis cuantitativo:

Tabla 2. Análisis cuantitativo (elaboración propia)

\begin{tabular}{|c|c|c|c|c|}
\hline \multicolumn{5}{|c|}{ Análisis cuantitativo } \\
\hline Nunca & Casi nunca & A veces & Casi siempre & Siempre \\
\hline $0 /$ no & 1 & 2 & 3 & $4 /$ sí \\
\hline
\end{tabular}


Respecto al análisis cualitativo, basado en la escala numérica empleada para el procedimiento anterior, se asocia a los siguientes colores:

Tabla 3. Análisis cualitativo (elaboración propia)

\begin{tabular}{|c|c|c|c|c|}
\hline \multicolumn{5}{|c|}{ Análisis cualitativo } \\
\hline Nunca & Casi nunca & A veces & Casi siempre & Siempre \\
\hline $0 /$ no & 1 & 2 & 3 & $4 /$ sí \\
\hline
\end{tabular}

De este modo, al realizar el estudio de cada uno de los manuales de texto, se llevan a cabo ambos análisis. Los valores puntuables se sitúan de forma vertical para poder rellenar — de forma horizontalcada pregunta del cuestionario con el número que le corresponde a la columna rellenada.

\subsection{Cuestionario}

El cuestionario que se presenta a continuación está compuesto por doce preguntas que pretenden desvelar los aspectos más relevantes expuestos en los primeros capítulos de este trabajo.

- Las preguntas 1 y 2 se dirigen a aquellos aspectos más generales que conciernen a la enseñanza de las locuciones dentro de la Programación.

- Las preguntas 3,4 y 5 se centran en conocer qué Contenidos se ofrecen y con qué categorías gramaticales se relacionan.

- Las preguntas $6,7,8$ y 9 estudian cómo se tratan estas unidades en las Actividades y con qué finalidad se emplean.

- Las últimas preguntas, de la 10 a la 12 , se destinan a averiguar si se promueve la Producción de textos y su uso contextualizado.

Veamos ahora el modelo que nos ha servido de plantilla para el análisis: 
Tabla 4. Cuestionario para el análisis (basado en el cuestionario de Blabia 2017)

\begin{tabular}{|c|c|c|c|c|c|c|}
\hline \multirow{2}{*}{\multicolumn{2}{|c|}{$\begin{array}{l}\text { Cuestionario para el análisis de manuales de } \\
\text { texto de Lengua y Literatura Castellana }\end{array}$}} & \multicolumn{5}{|c|}{ Valores puntuables } \\
\hline & & \multirow{2}{*}{$\begin{array}{l}\text { No/ } \\
\text { Nunca }\end{array}$} & \multirow{2}{*}{$\begin{array}{l}\text { Casi } \\
\text { nunca }\end{array}$} & \multirow[t]{2}{*}{ A veces } & \multirow{2}{*}{$\begin{array}{l}\text { Casi } \\
\text { siempre }\end{array}$} & \multirow{2}{*}{$\begin{array}{l}\text { Sí/ } \\
\text { Siempre }\end{array}$} \\
\hline 1 & $\begin{array}{l}\text { Programación: ¿La unidad didáctica en } \\
\text { la que se trabajan las locuciones } \\
\text { presenta una programación horizontal? }\end{array}$ & & & & & \\
\hline 2 & $\begin{array}{l}\text { Programación: ¿Las locuciones están } \\
\text { trabajadas dentro del apartado de } \\
\text { Gramática o Conocimiento de la lengua? }\end{array}$ & & & & & \\
\hline 3 & $\begin{array}{l}\text { Contenidos: ¿Son explicadas como parte } \\
\text { de las categorías gramaticales a las que } \\
\text { hacen referencia? }\end{array}$ & & & & & \\
\hline 4 & $\begin{array}{l}\text { Contenidos: ¿Se ofrece una definición } \\
\text { clara y detallada del concepto de } \\
\text { locución? }\end{array}$ & & & & & \\
\hline 5 & $\begin{array}{l}\text { Contenidos: ¿Se enumeran todas las } \\
\text { clases de locuciones que existen en la } \\
\text { lengua española? }\end{array}$ & & & & & \\
\hline 6 & $\begin{array}{l}\text { Contenidos: ¿Se utilizan ejemplos de } \\
\text { cada tipo para aclarar la definición? }\end{array}$ & & & & & \\
\hline 7 & $\begin{array}{l}\text { Actividades: ¿Aparecen incluidas en } \\
\text { actividades de análisis y comprensión? }\end{array}$ & & & & & \\
\hline 8 & $\begin{array}{l}\text { Actividades: ¿Aparecen incluidas en } \\
\text { actividades de producción? }\end{array}$ & & & & & \\
\hline 9 & $\begin{array}{l}\text { Actividades: ¿Están contextualizadas en } \\
\text { un texto? }\end{array}$ & & & & & \\
\hline 10 & $\begin{array}{l}\text { Producción: ¿Se enseña a utilizarlas en } \\
\text { contextos específicos? }\end{array}$ & & & & & \\
\hline 11 & $\begin{array}{l}\text { Producción: ¿Se promueve su uso en la } \\
\text { producción de textos escritos? }\end{array}$ & & & & & \\
\hline 12 & $\begin{array}{l}\text { Producción: ¿Se promueve su uso en la } \\
\text { producción de textos orales? }\end{array}$ & & & & & \\
\hline
\end{tabular}

Como se ha explicado anteriormente, este es el modelo elaborado para el estudio de los manuales. En él podemos observar tanto el análisis cuantitativo (numérico) como el cualitativo (con colores) que se realiza en cada una de las preguntas.

\section{Resultados}

El análisis de los manuales presentados en el apartado anterior nos ha permitido observar el tratamiento tradicional y escaso que reciben las locuciones en la mayoría de las preguntas presentadas.

Veamos, a partir de la siguiente tabla, cuáles han sido los resultados: 
Tabla 5. Análisis de los resultados

\begin{tabular}{|l|l|l|l|l|l|l|l|l|l|l|l|l|l|}
\hline MANUAL & 1 & 2 & 3 & 4 & 5 & 6 & 7 & 8 & 9 & 10 & 11 & 12 & Total \\
\hline Teide & 0 & 4 & 0 & 4 & 0 & 4 & 3 & 2 & 0 & 0 & 0 & 0 & 17 \\
\hline Sansy & 4 & 4 & 0 & 4 & 4 & 4 & 0 & 0 & 0 & 0 & 0 & 0 & 20 \\
\hline Anaya & 0 & 4 & 4 & 2 & 2 & 4 & 3 & 3 & 2 & 0 & 0 & 0 & 24 \\
\hline Editex & 0 & 4 & 4 & 2 & 1 & 4 & 2 & 0 & 0 & 0 & 0 & 0 & 17 \\
\hline Casals & 4 & 4 & 2 & 2 & 1 & 3 & 2 & 2 & 2 & 0 & 0 & 0 & 22 \\
\hline Micomicona & 0 & 0 & 0 & 0 & 0 & 0 & 0 & 0 & 0 & 0 & 0 & 0 & 0 \\
\hline Oxford & 0 & 4 & 4 & 4 & 3 & 4 & 1 & 0 & 0 & 0 & 0 & 0 & 20 \\
\hline McGraw-Hill & 0 & 4 & 2 & 0 & 1 & 4 & 0 & 2 & 0 & 0 & 0 & 0 & 13 \\
\hline Castellnou & 0 & 4 & 2 & 0 & 1 & 4 & 1 & 0 & 0 & 0 & 0 & 0 & 12 \\
\hline Santillana & 0 & 0 & 0 & 0 & 0 & 0 & 0 & 0 & 0 & 0 & 0 & 0 & 0 \\
\hline Total & 8 & 32 & 18 & 18 & 13 & 31 & 12 & 9 & 4 & 0 & 0 & 0 & \\
\hline
\end{tabular}

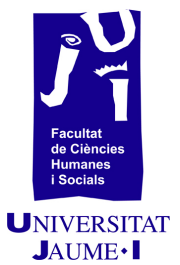

Escala de colores para la puntuación:

0 puntos: No/ Nunca

1 punto: Casi nunca

2 puntos: A veces

3 puntos: Casi siempre

4 puntos: Sí / Siempre

Como se puede observar en la tabla, si empezamos por la parte práctica, lo más destacado es la ausencia de tareas destinadas a la producción (preguntas 10, 11 y 12). En cuanto a las actividades (preguntas 7,8 y 9), estas se integran escasamente entre los ejercicios de las unidades donde se trabajan estas expresiones fijas. Por último, las primeras preguntas (de la 1 a la 6), de carácter más teórico, aluden a la Programación y Contenidos, y parece ser que sí ofrecen con mayor claridad la información que se pide. Incluso así, si nos fijamos en la primera pregunta - referida a la programación en horizontal-, veremos que la mayoría de los manuales carecen de este tipo de programación, la cual es necesaria para el desarrollo de un enfoque comunicativo dentro de las unidades.

\subsection{Tipo de programación}

La mayor parte de los manuales responden con un 'no' a la primera cuestión, referida a la estructuración en horizontal de la materia curricular. Aquellos que han contestado de forma negativa a esta cuestión distribuyen el contenido de la materia de forma vertical; en consecuencia, no se establece conexión entre el contenido lingüístico y el texto de la unidad. Consecuentemente, se observa una falta de contextualización y relación dentro de los textos y los recursos que en ellos aparecen. Así pues, la explicación de las funciones de las locuciones carece de sentido por no ofrecer una aplicación pragmática. 
Además, la disposición de la materia curricular está dividida en compartimentos estancos, separados y sin conexión alguna entre ellos, por lo que es casi imposible llevar un enfoque comunicativo a lo largo de estas programaciones y, por tanto, no se trabajan las locuciones contextualizadas.

La segunda cuestión consiste en conocer la ubicación de las locuciones dentro del Conocimiento de la lengua o, si el manual es más antiguo, en la Gramática. En los manuales más actuales hay más cohesión y sistematicidad a la hora de incluirlos dentro de este apartado, mientras que en aquellos que tienen una programación más tradicional, existe mayor variedad a la hora de situarlas entre Gramática y Léxico. Sin embargo, la clasificación en un apartado u otro no encuentra su correspondencia en las actividades, es decir, aquellos manuales que las sitúan en el apartado gramatical deberían hacer énfasis en la función de las locuciones como recursos argumentativos y de coherencia y cohesión, mientras que los manuales que las sitúan en el apartado del léxico tendrían que desarrollar con mayor amplitud sus funciones como recursos expresivos.

Incluso así, en este aspecto el resultado general es favorable, dado que la ubicación de la mayoría de ellas se recogen en el Conocimiento de la lengua $\mathrm{y}$, por consiguiente, esto posibilita el tratamiento de las locuciones en todas sus vertientes.

\subsection{Contenidos y actividades}

Las cuestiones de la 3 a la 6 pretenden indagar sobre cómo se ha establecido y explicado la base teórica de las locuciones.

La tercera cuestión se refiere a la inclusión de estas dentro de las categorías gramaticales a las que pertenecen: el proceso de análisis muestra de forma sorprendente las clasificaciones incompletas en la mayoría de los manuales.

En cuanto a la siguiente cuestión, referida a la definición del término locución (pregunta 4), siguen siendo la minoría los manuales que establecen las bases teóricas de forma clara para identificarlas y reconocer de qué tipo se trata. Consecuentemente, esta confusión concluye, como ya se ha comentado, en la presentación de una clasificación incompleta y poco objetiva.

Por último, la pregunta 6 se refiere al uso de ejemplos para mejorar la comprensión de las definiciones. En la mayoría de los casos, los resultados han sido afirmativos y ofrecen un listado de ejemplos que acompañan la definición.

En cuanto a las actividades, las cuestiones destinadas a ellas son las siguientes: 7,8 y 9.

La primera de ellas, la pregunta 7 , intenta descubrir si las actividades propuestas tienen como trasfondo el análisis y la comprensión de la teoría explicada anteriormente. Pero, como se 
observa en la tabla de resultados, son pocos los ejercicios que trabajan este aspecto.

A continuación, las siguientes actividades, la 8 y 9 , son prácticamente inexistentes en todos los manuales analizados. Como vemos en la tabla que resume los resultados, la mayoría de ellos responde de forma negativa a la motivación de promover actividades que desarrollen y potencien la producción de textos y ejercicios que impliquen una mínima contextualización de las locuciones.

\subsection{Producción}

Finalmente, de la cuestión 10 a la 12 se examina qué lugar se otorga a las locuciones dentro del texto cuando se proponen actividades de producción y si se promueve su uso como recursos lingüísticos en la elaboración de textos.

La cuestión número 10 pregunta: "¿Se enseña a utilizarlas en contextos específicos?». Todos los manuales responden con un 'no'. Consecuentemente, la intención didáctica que se esconde tras los contenidos queda incompleta por la ausencia de su aplicación en un contexto determinado. Esto también está ligado a la negación que se ha obtenido en la primera pregunta, es decir, si no se aplica una programación horizontal, las unidades probablemente carecen de un enfoque comunicativo y, en consecuencia, desaparece la motivación de la producción de textos, tanto orales -como se pregunta en la cuestión 11-, como escritos - cuestión 12-.

De estos datos se desprende que este es uno de los grandes aspectos que se debería fortalecer a la hora de incluir la enseñanza de las locuciones en un contexto según un enfoque comunicativo, dado que si solo se explica la teoría, pero no se trabajan los aspectos prácticos, el proceso de asimilación y comprensión de estos recursos lingüísticos queda incompleto.

\section{Conclusiones}

Tras el análisis de los manuales, se concluye que las locuciones son recursos lingüísticos que no se potencian en las clases de Secundaria y, en concreto, en 10 de Bachillerato.

El principal error recae en el tratamiento que reciben en los manuales, dado que se aíslan en cuadros situados en los laterales de las páginas como información secundaria $y$, además, nunca se relacionan con las actividades que se proponen en la unidad donde aparecen. Asimismo, no se presentan de forma sistemática y objetiva porque no se describen todas las subclases que se exponen en el presente trabajo.

Se quiere demostrar también que una mayor atención a las locuciones en la lengua materna de los alumnos posibilitaría un aumento de recursos lingüísticos a la hora de expresarse y, por tanto, 
una mayor competencia comunicativa. Es más, este proceso de

enseñanza que vincula las locuciones con otros elementos lingüísticos permite tanto la reflexión sobre el conocimiento de la lengua, como el desarrollo de las destrezas discursivas más inmediatas en su día a día.

\section{VIII.Bibliografía}

Belinchón Carmona, Mercedes. 1999. «Lenguaje no literal y aspectos pragmáticos de la comprensión». Psicolingüística del español, coordinado por Fernando Cuetos Vega y Manuel de Vega Rodríguez, 307-373. Madrid: Trotta.

Blabia Girau, Irene. 2017. ¿Usuarios o sabios de los textos periodísticos informativos?. Trabajo de Fin de Máster. Castelló de la Plana: Publicacions de la Universitat Jaume I.

Briz, Antonio. Grupo Val.Es.Co. 1995. "La conversación coloquial. Materiales para su estudio». Cuadernos de Filología 16: 175-189.

Bronckart, Jean Paul y Bernard Schneuwly. 1996. "La didáctica de la lengua materna: el nacimiento de una utopía indispensable». Textos de Didáctica de la Lengua y la Literatura 9: 61-79.

Coronas Peñarrocha, Ricardo. 2010. «Las oraciones coordinadas en los libros de texto de lengua castellana». En Libros de texto y enseñanza de la gramática, coordinado por Teresa Ribas, 117134. Barcelona: Graó.

Corpas Pastor, Gloria. 1996. Manual de fraseología español. Madrid: Gredos.

Coseriu, Eugenio. 1977. Estudios de lingüística románica. Madrid: Gredos.

Fontich, Xavier. 2014. «La enseñanza de la gramática en la educación secundaria». Didáctica de la Lengua y de la Literatura, Textos 67: 5-6.

Forment Fernández, Mạ del Mar. 1997. "La didáctica de la fraseología ayer y hoy: del aprendizaje memorístico al agrupamiento en los repertorios de funciones comunicativas». Centro Virtual Cervantes 8: 339-347.

García Muruais Ma Teresa. 1997. «Propuesta para la enseñanza de unidades fraseológicas en a clase de E/LE». Centro Virtual Cervantes 8: 363-369.

Gómez González, Alba y Clara Ureña Tormo. 2015. Locuciones y refranes para dar y toma. El libro para aprender más de 120 locuciones y refranes en español. Niveles B2 y C1. Alcalá de Henares: Publicaciones de la Universidad de Alcalá. 
Iliná, Natalia. 2000. «Fraseología española contemporánea: estado de la cuestión». Actas de la II Conferencia de hispanistas de Rusia, vol. 4, 207-218. Moscú: Facultad de Lenguas Extranjeras adjunta a la Universidad Estatal Lomonosov.

Lamas, Óscar Loureda. 1999. "Acerca del objeto y los objetivos de la enseñanza del Área de Lengua y Literatura en la Enseñanza Secundaria Obligatoria». Rilce 15 (2): 427-438.

Ley Orgánica 1105/2014, de 26 de diciembre, para la mejora de la calidad educativa. Boletín Oficial del Estado 3: 169-543.

Lomas, Carlos. 2003. "Aprender a comunicar(se) en las aulas». Ágora Digital 5: 1-17.

Martínez Marín, Juan. 2000. "Las unidades léxicas complejas en español: aspectos teóricos y descriptivos». Revista de Investigación Lingüística 3: 315-338.

Núñez-Román, Francisco. 2015. «Enseñar fraseología: consideraciones sobre la fraseodidáctica del español». Didáctica: Lengua y Literatura 27: 153-166.

Real Academia Española. 2010. Nueva gramática de la lengua española. Manual, Madrid: Espasa Calpe.

Ruiz Gurillo, Leonor. 2001. Las locuciones en el español actual. Madrid: Arcos.

Zuluaga, Alberto. 1980. «Introducción al estudio de las expresiones fijas». Studia Romanica et Lingüística 10: 126-141. 